\title{
Complex Permeability of LiZn/MnZn/PVC Composite Materials
}

\author{
R. Dosoudil*, M. Ušáková, A. Grusková and J. Sláma \\ Slovak University of Technology, Ilkovičova 3, 81219 Bratislava, Slovakia
}

\begin{abstract}
The effect of particle content, size, and the fraction ratio of the double LiZn/MnZn ferrite filler in hybrid $\mathrm{LiZn/MnZn/PVC} \mathrm{composites} \mathrm{on} \mathrm{the} \mathrm{frequency} \mathrm{dispersion} \mathrm{of} \mathrm{complex} \mathrm{permeability} \mathrm{has} \mathrm{been} \mathrm{studied} \mathrm{in} \mathrm{the}$ $1 \mathrm{MHz}-3 \mathrm{GHz}$ frequency range. The obtained relaxation type of permeability dispersion is due to the domain wall resonance, the natural ferromagnetic resonance, and the relaxation of magnetization rotation. The permeability and resonance/relaxation frequency of composites seem to be strongly affected by the change of particle content and fraction ratio of the double ferrite filler rather than by particle size.
\end{abstract}

PACS numbers: 72.80.Tm, 75.50.Gg, 76.50.+g

\section{Introduction}

Multicomponent composite materials with several magnetic fillers dispersed in a single polymeric matrix can have better magnetic properties (such as permeability) than composites with only one type of magnetic filler [1]. Especially those with more than one dispersion region in permeability spectra are a rare event. In this study, the relationship between structural factors such as particle content, size and the fraction ratio of the double ferrite filler in $\mathrm{LiZn} / \mathrm{MnZn} / \mathrm{PVC}$ composites and the material parameter such as complex permeability was investigated in the frequency range $1 \mathrm{MHz}^{-} 3 \mathrm{GHz}$. Also the contribution of two basic magnetizing mechanisms, namely the domain wall motion and the magnetic moment rotations in domains, has been analyzed.

\section{Experimental}

For purposes of this study, two types of spinel ferrites (in sintered and also powder form), i.e. a commercially available $\mathrm{Mn}_{0.52} \mathrm{Zn}_{0.43} \mathrm{Fe}_{2.05} \mathrm{O}_{4}$ and $\mathrm{Li}_{0.525} \mathrm{Zn}_{0.30} \mathrm{Ti}_{0.35} \mathrm{Fe}_{1.825} \mathrm{O}_{4}$ synthesized by a ceramic method at $1050{ }^{\circ} \mathrm{C} / 3.5 \mathrm{~h}$ in air were used. Composites were prepared by mixing the LiZn and MnZn ferrite powders with the polyvinylchloride (PVC) polymer matrix, followed by a low temperature hot pressing (at $135^{\circ} \mathrm{C}$ and $5 \mathrm{MPa}$ ). Three groups of composite materials (A, B, C) have been prepared: (A) constant particle size 0-250 $\mu \mathrm{m}$, constant fraction ratio of double LiZn:MnZn ferrite filler 0.5:0.5, and different particle contents 63 (A1), 53 (A2), 43 (A3) and 33 vol.\% (A4), (B) constant particle content $63 \mathrm{vol} \%$, constant fraction ratio of double LiZn:MnZn ferrite filler 0.5:0.5, and different particle sizes 0-250 (B1), 160-250 (B2), 80-160 (B3), 40-80 (B4) and 0-40 $\mu \mathrm{m}$ (B5), and (C) constant particle content 63 vol. $\%$, constant particle size $0-250 \mu \mathrm{m}$, and

\footnotetext{
* corresponding author; e-mail: rastislav.dosoudil@stuba.sk
}

different fraction ratios of dual LiZn:MnZn ferrite filler 0:1 (C1), 0.3:0.7 (C2), 0.5:0.5 (C3), 0.7:0.3 (C4) and 1:0 (C5). Multifrequency measurements of complex permeability $\tilde{\mu}=\mu^{\prime}-\mathrm{j} \mu^{\prime \prime}$ of samples have been performed by impedance spectroscopy $[2,3]$.

\section{Results and discussion}

Complex permeability versus frequency curves for sintered ferrites (LiZn and MnZn) are plotted in Fig. 1. The obtained resonance type of frequency dispersion of $\tilde{\mu}$ is caused by [2, 3]: (a) the domain wall resonance, (b) the natural ferromagnetic resonance or the forced precession of magnetic moments in domains, and (c) the relaxation of magnetization rotation. The second dispersion region observed in the case of LiZn ferrite has a critical (resonance/relaxation) frequency of about $719 \mathrm{MHz}$ and is attributed to the natural ferromagnetic resonance. The first dispersion frequency $(\approx 16 \mathrm{MHz})$ corresponds to the domain wall resonance and the relaxation of magnetization rotation. In case of $\mathrm{MnZn}$ ferrite, only one dispersion range is observed and is due to the coincidence of all dispersion ranges.

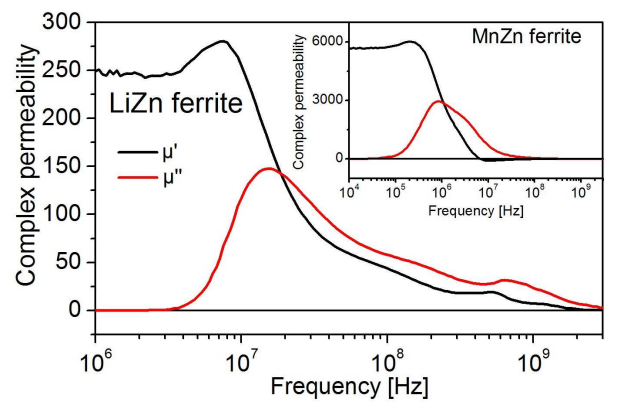

Fig. 1. Frequency dependences of complex permeability for sintered LiZn and MnZn (inset) ferrites.

Complex permeability versus frequency curves for composites with different particle content are depicted in 
Fig. 2a. The complex permeability $\tilde{\mu}$ showed the relaxation type of frequency dispersion. The value of $\mu^{\prime}$ decreased with decreasing filler particle content in the low frequency range below $20 \mathrm{MHz}$ due to the decrease of filler particle amount. The lower critical frequency $f_{\mathrm{c} 1}$, at which $\mu^{\prime \prime}$ has the first maximum value, raised with decreasing particle amount from about 247 to $405 \mathrm{MHz}$. The higher critical frequency $f_{\mathrm{c} 2}$, at which $\mu^{\prime \prime}$ has the second maximum value, corresponds to the second dispersion frequency for LiZn ferrite ( $\approx 719 \mathrm{MHz}$, Fig. 1).

The effect of particle size on permeability is shown in Fig. 2b. The $\mu^{\prime}$ decreased as the particle size decreased in the low frequency region from 22.5 (for sample B1) to 18.3 (for sample B5). The lower critical frequency $f_{\mathrm{c} 1}$ increased with decreasing particle size from about 247 to $312 \mathrm{MHz}$. The second maximum of $\mu^{\prime \prime}$ and the corresponding dispersion frequency (which is almost the same for all composites B1-B5: $\approx 719 \mathrm{MHz}$ ) is also present. The influence of particle size on $\mu^{\prime}$ and $f_{\mathrm{c} 1}$ is less than particle content.

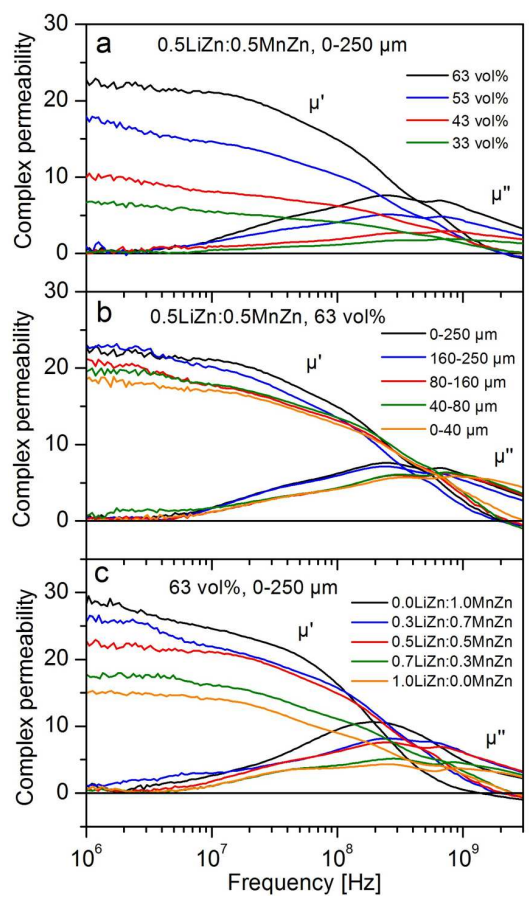

Fig. 2. Frequency dependences of complex permeability for composites with different (a) particle contents, (b) particle sizes, and (c) fraction ratios of double LiZn/ MnZn ferrite filler.

From Fig. 2c it follows that the permeability and its frequency dispersion changed continuously with the change of filler fraction ratio between two types of ferrite fillers. The value of $\mu^{\prime}$ increased from about 15.1 for sample C5 (two-component LiZn/PVC composite) to 28.9 for sample C1 (two-component MnZn/PVC composite). In case of sample $\mathrm{C} 1$, only one dispersion range is observed and the $\mu^{\prime \prime}$ has a maximum value of about 12 at a frequency of $207 \mathrm{MHz}$. This is in a good agreement with our previous studies [2,3]. The samples C2-C4 have two dispersion regions (similar to those presented in Fig. 2a,b). In this case, the $f_{\mathrm{c} 1}$ achieved the following values: $224 \mathrm{MHz}(\mathrm{C} 2), 247 \mathrm{MHz}$ (C3), and $265 \mathrm{MHz}(\mathrm{C} 4)$. In the case of sample $\mathrm{C} 5$, three dispersion regions were obtained. The first one is present at $35 \mathrm{MHz}$ (near the first dispersion frequency $16 \mathrm{MHz}$ of LiZn ferrite, Fig. 1), the second at $298 \mathrm{MHz}$, and the third at $719 \mathrm{MHz}$ (corresponding to the second dispersion frequency of LiZn ferrite).

The features observed in composites (the anomalous broadening of the resonance band, the shift of resonance towards to higher frequencies, and a considerable decrease in permeability) are direct consequences of microscopic demagnetizing effects in the composite structure in connection with the particle arrangement $[2,3]$. The presence of demagnetizing field $H_{\mathrm{D}}$ reduces the total induced magnetic moment in a composite: the decrease of particle content and/or size reduces the $\mu^{\prime}$ and increases $f_{\mathrm{c}}$ due to the demagnetizing effects: $\mu^{\prime} \approx M_{\mathrm{s}} /\left(H_{\mathrm{A}}+H_{\mathrm{D}}\right)$ and $f_{\mathrm{c}} \approx \mu_{0} \gamma /\left[2 \pi\left(H_{\mathrm{A}}+H_{\mathrm{D}}\right)\right]$, with $M_{\mathrm{s}}$ the saturation magnetization, $H_{\mathrm{A}}$ the effective anisotropy field, $\mu_{0}$ the permeability of free space and $\gamma$ the gyromagnetic ratio. Moreover, when only the fraction ratio was varied (Fig. 2c), the value of $H_{\mathrm{A}}$ was also affected by the change of filler from MnZn to LiZn ferrite: the $\mu^{\prime}$ decreases and $f_{\mathrm{c}}$ shifts higher due to the contribution of magnetocrystalline anisotropy to the $H_{\mathrm{A}}$; the existence of two (or three) dispersion ranges especially in composites with larger amount of LiZn ferrite is probably due to the presence of all three analyzed resonance mechanisms.

\section{Conclusions}

We have studied the influence of particle content, size, and the fraction ratio of the double LiZn/MnZn ferrite filler in hybrid LiZn/MnZn/PVC composites on the frequency dispersion of permeability in the frequency region $1 \mathrm{MHz}-3 \mathrm{GHz}$ using impedance spectroscopy. The resonance type of permeability dispersion was observed in ferrites in contrast to the composites where only relaxation type was achieved. In addition, more than one dispersion region in permeability spectra was reached.

\section{Acknowledgments}

This work was supported by 1/0529/10 and 1/0575/09 grants of VEGA agency of Slovakia.

\section{References}

[1] T. Kasagi, T. Tsutaoka, K. Hatakeyama, J. Magn. Magn. Mater. 272-276, 2224 (2004).

[2] R. Dosoudil, M. Ušáková, J. Franek, A. Grusková, J. Sláma, J. Magn. Magn. Mater. 320, e849 (2008).

[3] R. Dosoudil, M. Ušáková, J. Franek, J. Sláma, A. Grusková, IEEE Trans. Magn. 46, 436 (2010). 